\title{
Eigenvalue estimates for singular left-definite Sturm-Liouville operators
}

\author{
Jussi Behrndt, Roland Möws, and Carsten Trunk ${ }^{1}$
}

\begin{abstract}
The spectral properties of a singular left-definite Sturm-Liouville operator $J A$ are investigated and described via the properties of the corresponding right-definite selfadjoint counterpart $A$ which is obtained by substituting the indefinite weight function by its absolute value. The spectrum of the $J$-selfadjoint operator $J A$ is real and it follows that an interval $(a, b) \subset \mathbb{R}^{+}$is a gap in the essential spectrum of $A$ if and only if both intervals $(-b,-a)$ and $(a, b)$ are gaps in the essential spectrum of the $J$-selfadjoint operator $J A$. As one of the main results it is shown that the number of eigenvalues of $J A$ in $(-b,-a) \cup(a, b)$ differs at most by three from the number of eigenvalues of $A$ in the gap $(a, b)$; as a byproduct results on the accumulation of eigenvalues of singular left-definite Sturm-Liouville operators are obtained. Furthermore, left-definite problems with symmetric and periodic coefficients are treated, and several examples are included to illustrate the general results.
\end{abstract}

Mathematics Subject Classification (2010). Primary 34B24, 34L15; Secondary 47A10, 47E05.

Keywords. Sturm-Liouville operator, left-definite, eigenvalue estimate, spectral gap, essential spectrum, Titchmarsh-Weyl function.

\section{Introduction}

We investigate spectral properties of a Sturm-Liouville differential operator associated with the differential expression

$$
\tau=\frac{1}{r}\left(-\frac{d}{d x} p \frac{d}{d x}+q\right), \quad r, p^{-1}, q \in L_{\text {loc }}^{1}(\mathbb{R}) \text { real, } p>0 \text { a.e. }
$$

In contrast to standard Sturm-Liouville theory we do not assume that the weight function $r$ is positive. Instead we consider indefinite Sturm-Liouville operators and differential expressions; here it will be assumed that there exists some $c \in \mathbb{R}$ such that the weight function $r$ is positive on $(c, \infty)$ and negative on $(-\infty, c)$. Suppose

\footnotetext{
${ }^{1}$ The authors thank Gerald Teschl for fruitful remarks.
} 
that the corresponding definite differential expression

$$
\ell=\frac{1}{|r|}\left(-\frac{d}{d x} p \frac{d}{d x}+q\right)
$$

is in the limit point case at both singular endpoints $-\infty$ and $\infty$, or, equivalently, that the maximal differential operator $A$ associated with $\ell$ in the weighted Hilbert space $L_{|r|}^{2}(\mathbb{R})$ is selfadjoint. If $J$ denotes the multiplication by $\operatorname{sgn} r$, then formally the indefinite and definite differential expressions $\tau$ and $\ell$ are related via $\tau=J \ell$, and hence $J A$ is the maximal operator associated with $\tau$ in $L_{|r|}^{2}(\mathbb{R})$. Observe that the indefinite Sturm-Liouville operator $J A$ is neither symmetric nor selfadjoint in the Hilbert space $L_{|r|}^{2}(\mathbb{R})$ but $J A$ is selfadjoint with respect to an indefinite inner product (which has $J$ as its Gramian); we shall say that $J A$ is a $J$-selfadjoint operator in $L_{|r|}^{2}(\mathbb{R})$.

A modern topic in Sturm-Liouville theory is the study of qualitative and quantitative spectral properties of indefinite Sturm-Liouville differential operators. One of the standard approaches is to describe the spectrum $\sigma(J A)$ of the indefinite operator $J A$ via the selfadjoint operator $A$ and its spectral properties. In the left-definite case, i.e. $\min \sigma(A)>0$, it follows that the spectrum of $J A$ is real with a gap around 0 and accumulates to $+\infty$ and $-\infty$, see, e.g., [8], [21], [30], and [1], [7] for corresponding abstract results. If $A$ is semibounded from below and the essential spectrum satisfies $\min \sigma_{\mathrm{ess}}(A)>0$, then the nonreal spectrum of $J A$ consists of at most finitely many eigenvalues, the essential spectrum $\sigma_{\text {ess }}(J A)$ is real with a gap around 0 , and $\sigma(J A) \cap \mathbb{R}$ accumulates to $+\infty$ and $-\infty$, see, e.g., [8], [25], and [20]. The spectral analysis of $J A$ in the case $\min \sigma_{\text {ess }}(A) \leq 0$ is more difficult; we refer to [2], [4], and [17] for more details and to [3], [5], [9], [10], [15], and [16] for related questions and further references.

The main objective of the present paper is to prove a local estimate on the number of eigenvalues of $J A$ in terms of the number of eigenvalues of $A$ in gaps of the essential spectrum in the left-definite case, i.e. $\min \sigma(A)>0$. In this situation it is not difficult to see that for $0 \leq a<b$ we have

$$
(a, b) \cap \sigma_{\mathrm{ess}}(A)=\varnothing \quad \text { if and only if } \quad((-b,-a) \cup(a, b)) \cap \sigma_{\mathrm{ess}}(J A)=\emptyset .
$$

Our main result Theorem 4.1 reads as follows: if $(a, b) \cap \sigma_{\text {ess }}(A)=\varnothing$, then the number of eigenvalues $n_{A}(a, b)$ of $A$ in $(a, b)$ differs at most by three from the number $n_{J A}(-b,-a)+n_{J A}(a, b)$ of eigenvalues of $J A$ in $(-b,-a) \cup(a, b)$,

$$
\left|n_{A}(a, b)-\left(n_{J A}(-b,-a)+n_{J A}(a, b)\right)\right| \leq 3 \text {. }
$$

Under the assumption that the coefficients $p, q$, and $r$ are symmetric with respect to 0 the estimate on the number of eigenvalues is improved in Theorem 4.4 for intervals $(a, b)$ with the property $0 \leq a<\min \sigma(A)<b \leq \min \sigma_{\text {ess }}(A)$. The above estimates also yield results on accumulation properties of eigenvalues of $J A$. More precisely, if, e.g., $b \in \sigma_{\text {ess }}(A)$ and the eigenvalues of $A$ in $(a, b)$ accumulate 
to $b$, then the eigenvalues of $J A$ in the gaps $(-b,-a)$ and $(a, b)$ of the essential spectrum accumulate to $-b$ or $b$. This allows to transfer results on the accumulation (or non-accumulation) of eigenvalues to the boundary of the essential spectrum of definite Sturm-Liouville operators (as, e.g., the classical Kneser criterion from [19] or recent extensions of it in [12], [13], [23], and [24]) into the left-definite setting; see Section 5.1 for more details.

The paper is organized as follows. In Section 2 the operators $A, J A$, and the Dirichlet operators associated with the restrictions $\ell_{+}$and $\ell_{-}$of the definite differential expression $\ell$ onto $(c, \infty)$ and $(-\infty, c)$ are introduced and some simple properties of their spectra and essential spectra are collected. Section 3 establishes the connection of the poles and zeros of the Titchmarsh-Weyl coefficients $m_{+}, m_{-}$associated with $\ell_{+}$and $\ell_{-}$, respectively, with the poles and zeros of the Titchmarsh-Weyl coefficient $M$ associated with $\tau$. This connection is then used to describe the isolated eigenvalues of $A$ and $J A$ in terms of the poles and zeros of the functions $m_{+}, m_{-}$ and $M$. The representation of the function $M$ in terms of a Nevanlinna function in Proposition 3.4 and the corresponding monotonicity properties are the crucial ingredients in the proofs of our main results Theorem 4.1 and Theorem 4.4 in Section 4. In Section 5 Kneser's criterion is applied in the left-definite setting and the general results are illustrated in this situation. Furthermore, a class of periodic problems is considered (see also [22], [26] and [30], §12.8, for a slightly different indefinite periodic situation), and a simple solvable problem is briefly discussed.

\section{Preliminaries on definite and indefinite Sturm-Liouville operators}

Let $r, p^{-1}, q \in L_{\mathrm{loc}}^{1}(\mathbb{R})$ be real valued functions with $p>0$ and $r \neq 0$ almost everywhere. We consider the differential expressions

$$
\tau=\frac{1}{r}\left(-\frac{d}{d x} p \frac{d}{d x}+q\right) \quad \text { and } \quad \ell=\frac{1}{|r|}\left(-\frac{d}{d x} p \frac{d}{d x}+q\right)
$$

from (1.1) and (1.2). In this section we collect some simple properties on the spectra of the associated maximal operators. It is assumed that the following condition (I) holds for the weight function $r$ :

(I) There exists $c \in \mathbb{R}$ such that the restriction $r_{+}=r \uparrow_{(c, \infty)}$ is positive almost everywhere and the restriction $r_{-}=r \uparrow_{(-\infty, c)}$ is negative almost everywhere.

The restrictions of the functions $p$ and $q$ onto the intervals $(c, \infty)$ and $(-\infty, c)$ will be denoted by $p_{+}, q_{+}$and $p_{-}, q_{-}$, respectively.

The space of all (equivalence classes of) complex valued measurable functions $f$ such that $|f|^{2}|r| \in L^{1}(\mathbb{R})$ is denoted by $L_{|r|}^{2}(\mathbb{R})$. Equipped with the scalar product

$$
(f, g)=\int_{\mathbb{R}} f(x) \overline{g(x)}|r(x)| d x, \quad f, g \in L_{|r|}^{2}(\mathbb{R}),
$$


this space is a Hilbert space. The maximal operator $A f=\ell f$ associated with the definite Sturm-Liouville expression $\ell$ in $L_{|r|}^{2}(\mathbb{R})$ is defined on the dense subspace

$$
\mathfrak{D}=\left\{f \in L_{|r|}^{2}(\mathbb{R}): f, p f^{\prime} \text { locally absolutely continuous, } \ell f \in L_{|r|}^{2}(\mathbb{R})\right\} .
$$

We denote by $\mathfrak{D}_{+}$and $\mathfrak{D}_{-}$the spaces of functions on $(c, \infty)$ and $(-\infty, c)$ which are restrictions of functions from $\mathfrak{D}$ onto $(c, \infty)$ and $(-\infty, c)$, respectively. Throughout this paper it will be assumed that $A$ satisfies the following condition (II):

(II) The maximal operator $A f=\ell f$ defined on $\operatorname{dom} A=\mathfrak{D}$ is selfadjoint in $L_{|r|}^{2}(\mathbb{R})$ and $\min \sigma(A)>0$ holds.

Recall that $A$ is selfadjoint if and only if the definite Sturm-Liouville expression $\ell$ is in the limit point case at both singular endpoints $+\infty$ and $-\infty$.

Besides the definite inner product $(\cdot, \cdot)$ in $(2.1)$ the space $L_{|r|}^{2}(\mathbb{R})$ will also be equipped with the indefinite inner product $[\cdot, \cdot]$ defined by

$$
[f, g]=\int_{\mathbb{R}} f(x) \overline{g(x)} r(x) d x, \quad f, g \in L_{|r|}^{2}(\mathbb{R}) .
$$

The space $L_{r}^{2}(\mathbb{R})=\left(L_{|r|}^{2}(\mathbb{R}),[\cdot, \cdot]\right)$ is a Krein space, the inner products $(\cdot, \cdot)$ and $[\cdot, \cdot]$ are connected via the fundamental symmetry $(J f)(x)=\operatorname{sgn}(r(x)) f(x), x \in \mathbb{R}$, that is, the relations

$$
(J f, g)=[f, g] \quad \text { and }[f, g]=(J f, g), \quad f, g \in L_{|r|}^{2}(\mathbb{R}),
$$

hold, see, e.g., [1] and [7]. Note that formally we have $\tau=J \ell$. The maximal operator associated with $\tau$ coincides with $J A$. This operator is selfadjoint with respect to the indefinite inner product [., $\cdot]$; we shall say that $J A$ is $J$-selfadjoint in the Hilbert space $L_{|r|}^{2}(\mathbb{R})$. As a consequence of condition (II) and well-known properties of $J$-nonnegative operators (see, e.g., [7]) we obtain the next proposition.

Proposition 2.1. Assume that conditions (I) and (II) hold. Then the indefinite SturmLiouville operator

$$
J A f=\tau f=\frac{1}{r}\left(-\left(p f^{\prime}\right)^{\prime}+q f\right), \quad f \in \operatorname{dom} J A=\mathfrak{D},
$$

is a $J$-selfadjoint operator in $L_{|r|}^{2}(\mathbb{R})$ with

$$
\sigma(J A) \subset \mathbb{R} \text { and } 0 \in \rho(J A) .
$$

Each eigenvalue $\lambda$ of $J A$ is simple, i.e., $\operatorname{dim} \operatorname{ker}(J A-\lambda)=1$ and there is no Jordan chain of length greater than one. 
For a more detailed analysis of the spectrum of $J A$ it is useful to consider the (definite) differential expressions

$$
\ell_{+}=\frac{1}{r_{+}}\left(-\frac{d}{d x} p_{+} \frac{d}{d x}+q_{+}\right) \quad \text { and } \quad \ell_{-}=-\frac{1}{r_{-}}\left(-\frac{d}{d x} p_{-} \frac{d}{d x}+q_{-}\right)
$$

and the associated differential operators in the subspaces $L_{r_{+}}^{2}(c, \infty)$ and $L_{-r_{-}}^{2}(-\infty, c)$ which consist of restrictions of functions from $L_{|r|}^{2}(\mathbb{R})$ onto the intervals $(c, \infty)$ and $(-\infty, c)$, respectively. It follows from condition (I) that $L_{r_{+}}^{2}(c, \infty)$ and $L_{-r_{-}}^{2}(-\infty, c)$ equipped with

$$
\begin{aligned}
& \left(h_{1}, h_{2}\right)_{+}=\int_{c}^{\infty} h_{1}(x) \overline{h_{2}(x)} r_{+}(x) d x, \quad h_{1}, h_{2} \in L_{r_{+}}^{2}(c, \infty), \\
& \left(k_{1}, k_{2}\right)_{-}=\int_{-\infty}^{c} k_{1}(x) \overline{k_{2}(x)}\left(-r_{-}(x)\right) d x, \quad k_{1}, k_{2} \in L_{-r_{-}}^{2}(-\infty, c),
\end{aligned}
$$

are Hilbert spaces. Since $\ell$ is in the limit point case at $+\infty$ and $-\infty$ it follows that the (restricted) differential expressions $\ell_{+}$and $\ell_{-}$are in the limit point case at $+\infty$ and $-\infty$, respectively, and regular at $c$. In Section 3 below we will make use of the Lagrange identities

$$
\begin{aligned}
\left(\ell_{+} h_{1}, h_{2}\right)_{+}-\left(h_{1}, \ell_{+} h_{2}\right)_{+} & =\left(p_{+} h_{1}^{\prime}\right)(c) \overline{h_{2}(c)}-h_{1}(c) \overline{\left(p_{+} h_{2}^{\prime}\right)(c)}, \\
\left(\ell_{-} k_{1}, k_{2}\right)_{-}-\left(k_{1}, \ell_{-} k_{2}\right)_{-} & =-\left(p_{-} k_{1}^{\prime}\right)(c) \overline{k_{2}(c)}+k_{1}(c) \overline{\left(p_{-} k_{2}^{\prime}\right)(c),}
\end{aligned}
$$

which hold for all $h_{1}, h_{2} \in \mathfrak{D}_{+}$and $k_{1}, k_{2} \in \mathfrak{D}_{-}$. The Dirichlet operators

$$
\begin{aligned}
& B_{+} h=\ell_{+} h, \quad \operatorname{dom} B_{+}=\left\{h \in \mathfrak{D}_{+}: h(c)=0\right\}, \\
& B_{-} k=\ell_{-} k, \quad \operatorname{dom} B_{-}=\left\{k \in \mathfrak{D}_{-}: k(c)=0\right\},
\end{aligned}
$$

associated with $\ell_{+}$and $\ell_{-}$in (2.2) are selfadjoint in the Hilbert spaces $L_{r_{+}}^{2}(c, \infty)$ and $L_{-r_{-}}^{2}(-\infty, c)$, respectively. Then the orthogonal sums $B=B_{+} \oplus B_{-}$and $J B=B_{+} \oplus\left(-B_{-}\right)$are selfadjoint operators in $L_{|r|}^{2}(\mathbb{R})$. The next lemma on the spectrum and essential spectrum of the selfadjoint operators $A, B$, and $B_{ \pm}$will be useful later on. For a closed operator $T$ in a Hilbert space the essential spectrum $\sigma_{\text {ess }}(T)$ consists of all $\lambda \in \mathbb{C}$ such that $T-\lambda$ is not a Fredholm operator. Note that for a selfadjoint operator or a $J$-nonnegative operator $T$ with $\rho(T) \neq \varnothing$ the set $\sigma_{\text {ess }}(T)$ coincides with those spectral points which are no isolated eigenvalues of finite multiplicity.

Lemma 2.2. Assume that conditions (I) and (II) are satisfied. For the spectra of the operators $A, B$ and $B_{ \pm}$the following relations hold:

(i) $\min \sigma(A) \leq \min \sigma(B)$ and $\min \sigma(A) \leq \min \sigma\left(B_{ \pm}\right)$;

(ii) $\sigma_{\mathrm{ess}}(A)=\sigma_{\mathrm{ess}}\left(B_{+}\right) \cup \sigma_{\mathrm{ess}}\left(B_{-}\right)=\sigma_{\mathrm{ess}}(B)$ and $\sigma_{\mathrm{ess}}\left(B_{ \pm}\right) \subset \sigma_{\mathrm{ess}}(A)$; 
(iii) $\min \sigma_{\mathrm{ess}}(A)=\min \left\{\min \sigma_{\mathrm{ess}}\left(B_{+}\right), \min \sigma_{\mathrm{ess}}\left(B_{-}\right)\right\}=\min \sigma_{\mathrm{ess}}(B)$.

(iv) Denote by $E_{A}$ and $E_{B}$ the spectral functions of $A$ and $B$, respectively. For an open interval $\Delta$ with $\Delta \cap \sigma_{\mathrm{ess}}(A)=\emptyset$ the estimate

$$
\left|\operatorname{dim} \operatorname{ran} E_{A}(\Delta)-\operatorname{dim} \operatorname{ran} E_{B}(\Delta)\right| \leq 1
$$

holds if the corresponding quantities are finite. Otherwise $\operatorname{dim} \operatorname{ran} E_{A}(\Delta)=\infty$ if and only if dim $\operatorname{ran} E_{B}(\Delta)=\infty$.

Observe that the case $\operatorname{dim} \operatorname{ran} E_{A}(\Delta)=\operatorname{dim} \operatorname{ran} E_{B}(\Delta)=\infty$ can only occur if one or both of the endpoints of $\Delta$ belong to the essential spectrum of $A$.

Proof. (i) Define the closed symmetric operators $S_{+}$and $S_{-}$in the Hilbert spaces $L_{r_{+}}^{2}(c, \infty)$ and $L_{-r_{-}}^{2}(-\infty, c)$ by

$$
S_{+} h=\ell_{+} h, \quad \operatorname{dom} S_{+}=\left\{h \in \mathfrak{D}_{+}: h(c)=\left(p_{+} h^{\prime}\right)(c)=0\right\}
$$

and

$$
S_{-} k=\ell_{-} k, \quad \operatorname{dom} S_{-}=\left\{k \in \mathfrak{D}_{-}: k(c)=\left(p_{-} k^{\prime}\right)(c)=0\right\} .
$$

As the orthogonal sum $S_{+} \oplus S_{-}$is a restriction of $A$ it follows that $S_{+} \oplus S_{-}$is a symmetric operator with a lower bound larger or equal to $\min \sigma(A)$ which is positive by condition (II). Clearly, also $S_{+}$and $S_{-}$are symmetric operators with lower bounds larger or equal to $\min \sigma(A)$. As $B_{+}$and $B_{-}$are the Friedrichs extensions of $S_{+}$and $S_{-}$(see [27], Theorem 3 and Corollary 2) also their lower bounds are larger or equal to $\min \sigma(A)$. This shows the second statement in (i); the first assertion in (i) is an immediate consequence.

The assertions in (ii) and (iii) follow from

$$
\operatorname{dim} \operatorname{ran}\left((B-\lambda)^{-1}-(A-\lambda)^{-1}\right)=1, \quad \lambda \in \rho(A) \cap \rho(B),
$$

whereas (2.5) itself is a consequence of the fact that $A$ and $B$ are selfadjoint extensions of the symmetric operator $R f=\ell f$, $\operatorname{dom} R=\{f \in \mathfrak{D}: f(c)=0\}$, which has defect numbers $(1,1)$. This together with [6], $\S 9.3$, Theorem 3 , implies (iv).

The following proposition on the essential spectrum of the indefinite SturmLiouville operator $J A$ complements the statements in Proposition 2.1. It is a simple consequence of Lemma 2.2 and $\operatorname{dim} \operatorname{ran}\left((J A-\lambda)^{-1}-(J B-\lambda)^{-1}\right)=1$ for all $\lambda \in \rho(J A) \cap \rho(J B)$. Note that $\rho(J A) \cap \rho(J B) \neq \varnothing$ by Proposition 2.1.

Proposition 2.3. Assume that conditions (I) and (II) hold. Then the essential spectrum of the indefinite Sturm-Liouville operator JA is given by

$$
\sigma_{\mathrm{ess}}(J A)=\sigma_{\mathrm{ess}}(J B)=\left(\sigma_{\mathrm{ess}}\left(B_{+}\right) \cup \sigma_{\mathrm{ess}}\left(-B_{-}\right)\right) \subset\left(\sigma_{\mathrm{ess}}(A) \cup \sigma_{\mathrm{ess}}(-A)\right) .
$$




\section{The function $M$}

In this section we define a function $M$ with the help of Titchmarsh-Weyl coefficients $m_{+}$and $m_{-}$associated with the differential expressions $\ell_{+}$and $\ell_{-}$in (2.2). Since it turns out that the zeros of $M$ coincide with the isolated eigenvalues of the indefinite Sturm-Liouville operator $J A$ we shall study the monotonicity properties of $M$, which then lead to eigenvalue estimates in the next section. As a byproduct we also obtain a result on the size of the spectral gap of $J A$ around zero in Proposition 3.3 below.

Assume throughout this section that conditions (I) and (II) hold and let $B_{+}$ and $B_{-}$be the selfadjoint Dirichlet operators in the Hilbert spaces $L_{r_{+}}^{2}(c, \infty)$ and $L_{-r_{-}}^{2}(-\infty, c)$ from (2.4), and let $\lambda \in \rho\left(B_{+}\right)$and $\mu \in \rho\left(B_{-}\right)$. As $\ell_{+}$and $\ell_{-}$are in the limit point case at $+\infty$ and at $-\infty$, respectively, there are unique (up to a constant multiple) solutions $h_{\lambda} \in \mathfrak{D}_{+}$and $k_{\mu} \in \mathfrak{D}_{-}$of the differential equations

$$
\ell_{+} h=\lambda h \quad \text { and } \quad \ell_{-} k=\mu k .
$$

The functions $m_{ \pm}: \rho\left(B_{ \pm}\right) \rightarrow \mathbb{C}$ are defined by

$$
m_{+}(\lambda)=\frac{\left(p_{+} h_{\lambda}^{\prime}\right)(c)}{h_{\lambda}(c)} \quad \text { and } \quad m_{-}(\mu)=\frac{\left(p_{-} k_{\mu}^{\prime}\right)(c)}{k_{\mu}(c)} .
$$

It is obvious that the poles of $m_{ \pm}$coincide with the isolated eigenvalues of $B_{ \pm}$and that the poles of the function $\lambda \mapsto m_{-}(-\lambda)$ coincide with the isolated eigenvalues of $-B_{-}$. The functions $m_{ \pm}$are holomorphic on $\rho\left(B_{ \pm}\right)$, they do not admit analytic extensions to points of $\sigma\left(B_{ \pm}\right)$, and they are symmetric with respect to the real axis, i.e.

$$
m_{+}(\bar{\lambda})=\overline{m_{+}(\lambda)} \quad \text { and } \quad m_{-}(\bar{\mu})=\overline{m_{-}(\mu)} .
$$

If we fix solutions $h_{\lambda}$ and $k_{\mu}$ with $h_{\lambda}(c)=1$ and $k_{\mu}(c)=1$ it follows from (2.3) that the relations

$$
\begin{aligned}
& (\lambda-\bar{\lambda})\left(h_{\lambda}, h_{\lambda}\right)_{+}=\left(\ell_{+} h_{\lambda}, h_{\lambda}\right)_{+}-\left(h_{\lambda}, \ell_{+} h_{\lambda}\right)_{+}=m_{+}(\lambda)-\overline{m_{+}(\lambda)}, \\
& (\bar{\mu}-\mu)\left(k_{\mu}, k_{\mu}\right)_{-}=\left(k_{\mu}, \ell_{-} k_{\mu}\right)_{-}-\left(\ell_{-} k_{\mu}, k_{\mu}\right)_{-}=m_{-}(\mu)-\overline{m_{-}(\mu)},
\end{aligned}
$$

hold. Therefore, $\pm m_{ \pm}$are so-called Nevanlinna functions. Recall that a complexvalued function $N$ is said to be a Nevanlinna function if $N$ is holomorphic on $\mathbb{C} \backslash \mathbb{R}$ and the properties

$$
N(\bar{\lambda})=\overline{N(\lambda)} \text { and } \quad \frac{\operatorname{Im} N(\lambda)}{\operatorname{Im} \lambda} \geq 0
$$

hold for all $\lambda \in \mathbb{C} \backslash \mathbb{R}$. For later purposes it is important to note that a Nevanlinna function $N$ is monotone increasing on real intervals which belong to its domain of holomorphy and that $N$ is equal to a constant on such an interval if and only if $N$ is a constant function on $\mathbb{C}$, see, e.g., [18].

In the following we will relate the zeros and poles of the function

$$
M(\lambda)=m_{+}(\lambda)-m_{-}(-\lambda), \quad \lambda \in \rho\left(B_{+}\right) \cap \rho\left(-B_{-}\right),
$$


with the eigenvalues of the operators $J A$ and $J B$. Clearly, the domain of holomorphy of $M$ contains the interval $\left(-\min \sigma\left(B_{-}\right), \min \sigma\left(B_{+}\right)\right)$, the poles of $M$ in $\left[\min \sigma\left(B_{+}\right), \infty\right)$ and $\left(-\infty,-\min \sigma\left(B_{-}\right)\right]$coincide with the poles of $\lambda \mapsto m_{+}(\lambda)$ and $\lambda \mapsto m_{-}(-\lambda)$, respectively. Hence each pole of $M$ in $\left[\min \sigma\left(B_{+}\right), \infty\right)$ is an isolated eigenvalue of $B_{+}$and each pole of $M$ in $\left(-\infty,-\min \sigma\left(B_{-}\right)\right]$is an isolated eigenvalue of $-B_{-}$. Therefore, each pole of $M$ is an isolated eigenvalue of the operator $J B=B_{+} \oplus\left(-B_{-}\right)$and, vice versa, every isolated eigenvalue of $J B$ is a pole of $M$. This shows assertion (ii) in the next proposition.

Proposition 3.1. For $\lambda \notin \sigma_{\mathrm{ess}}(J A)$ the following assertions hold:

(i) $\lambda \in \sigma_{p}(J A)$ if and only if $\lambda$ is a zero of $M$;

(ii) $\lambda \in \sigma_{p}(J B)$ if and only if $\lambda$ is a pole of $M$.

Proof. It remains to show assertion (i). For this observe first that $\lambda \notin \sigma_{\text {ess }}(J A)=$ $\sigma_{\text {ess }}\left(B_{+}\right) \cup \sigma_{\text {ess }}\left(-B_{-}\right)$is an eigenvalue of $J A$ with corresponding eigenfunction $f_{\lambda} \in$ $\mathfrak{D}$ if and only if $f_{\lambda}=h_{\lambda} \oplus k_{-\lambda}$, where $h_{\lambda} \in \mathfrak{D}_{+}$and $k_{-\lambda} \in \mathfrak{D}_{-}$are the (nontrivial) restrictions of $f_{\lambda}$ onto $(c, \infty)$ and $(-\infty, c)$, respectively, which satisfy the differential equations

$$
\ell_{+} h_{\lambda}=\lambda h_{\lambda}, \quad \ell_{-} k_{-\lambda}=-\lambda k_{-\lambda},
$$

and the conditions

$$
h_{\lambda}(c)=k_{-\lambda}(c), \quad\left(p_{+} h_{\lambda}^{\prime}\right)(c)=\left(p_{-} k_{-\lambda}^{\prime}\right)(c) .
$$

As a simple consequence we conclude

$$
\sigma_{p}(J A) \cap \sigma_{p}\left(B_{+}\right) \cap \rho\left(-B_{-}\right)=\varnothing \quad \text { and } \quad \sigma_{p}(J A) \cap \sigma_{p}\left(-B_{-}\right) \cap \rho\left(B_{+}\right)=\varnothing .
$$

Furthermore, $\sigma_{p}\left(B_{+}\right) \cap \sigma_{p}\left(-B_{-}\right)=\varnothing$ by Lemma 2.2(i) and condition (II) and, hence, it is sufficient to prove the equivalence in (i) for $\lambda \in \rho\left(B_{+}\right) \cap \rho\left(-B_{-}\right)$.

Assume first that $\lambda \in \sigma_{p}(J A) \cap \rho\left(B_{+}\right) \cap \rho\left(-B_{-}\right)$, so that (3.2) and (3.3) hold for some corresponding eigenfunction $f_{\lambda}=h_{\lambda} \oplus k_{-\lambda}$ of $J A$ and $h_{\lambda}(c)=k_{-\lambda}(c) \neq 0$. This yields

$$
m_{+}(\lambda)=\frac{\left(p_{+} h_{\lambda}^{\prime}\right)(c)}{h_{\lambda}(c)}=\frac{\left(p_{-} k_{-\lambda}^{\prime}\right)(c)}{k_{-\lambda}(c)}=m_{-}(-\lambda)
$$

and hence $M(\lambda)=0$. Conversely, let $\lambda \in \rho\left(B_{+}\right) \cap \rho\left(-B_{-}\right)$be a zero of $M$ and let $h_{\lambda} \in \mathfrak{D}_{+}$and $k_{-\lambda} \in \mathfrak{D}_{-}$be the (nontrivial) solutions of (3.2) which satisfy $h_{\lambda}(c)=k_{-\lambda}(c) \neq 0$. From $M(\lambda)=0$ we obtain $m_{+}(\lambda)=m_{-}(-\lambda)$ and it follows from (3.4) that also the second condition in (3.3) is satisfied by $h_{\lambda}$ and $k_{-\lambda}$. Therefore $f_{\lambda}=h_{\lambda} \oplus k_{-\lambda}$ belongs to $\mathfrak{D}$ and is an eigenfunction of $J A$ corresponding to $\lambda$.

In a similar way as in Proposition 3.1 the eigenvalues of $A$ and of $B=B_{+} \oplus B_{-}$ are related to the poles and zeros of the functions $m_{+}$and $m_{-}$. Since the isolated eigenvalues of $B_{+}$and $B_{-}$coincide with the poles of $m_{+}$and $m_{-}$it is clear that $\lambda$ is 
an eigenvalue of $B$ if and only if $\lambda$ is a pole of $m_{+}$or $m_{-}$; this shows item (ii) in the next proposition. For the convenience of the reader also the first item will be shown in detail.

Proposition 3.2. For $\lambda \notin \sigma_{\mathrm{ess}}(A)$ the following assertions hold:

(i) $\lambda \in \sigma_{p}(A)$ if and only if $\lambda$ is a either a zero of $m_{+}-m_{-}$or a pole of both $m_{+}$ and $m_{-}$;

(ii) $\lambda \in \sigma_{p}(B)$ if and only if $\lambda$ is a pole of $m_{+}$or of $m_{-}$.

Proof. It remains to show assertion (i). For this observe first that $\lambda \notin \sigma_{\text {ess }}(A)=$ $\sigma_{\text {ess }}\left(B_{+}\right) \cup \sigma_{\text {ess }}\left(B_{-}\right)$is an eigenvalue of $A$ with corresponding eigenfunction $f_{\lambda} \in \mathfrak{D}$ if and only if $f_{\lambda}=h_{\lambda} \oplus k_{\lambda}$, where $h_{\lambda} \in \mathfrak{D}_{+}$and $k_{\lambda} \in \mathfrak{D}_{-}$are the (nontrivial) restrictions of $f_{\lambda}$ onto $(c, \infty)$ and $(-\infty, c)$, respectively, which satisfy the differential equations

$$
\ell_{+} h_{\lambda}=\lambda h_{\lambda}, \quad \ell_{-} k_{\lambda}=\lambda k_{\lambda},
$$

and the conditions

$$
h_{\lambda}(c)=k_{\lambda}(c), \quad\left(p_{+} h_{\lambda}^{\prime}\right)(c)=\left(p_{-} k_{\lambda}^{\prime}\right)(c) .
$$

Hence

$$
\sigma_{p}(A) \cap \sigma_{p}\left(B_{+}\right) \cap \rho\left(B_{-}\right)=\emptyset \quad \text { and } \quad \sigma_{p}(A) \cap \sigma_{p}\left(B_{-}\right) \cap \rho\left(B_{+}\right)=\emptyset .
$$

Assume first that $\lambda$ is an eigenvalue of $A$. Then either $\lambda \in \rho\left(B_{+}\right) \cap \rho\left(B_{-}\right)$or $\lambda \in \sigma_{p}\left(B_{+}\right) \cap \sigma_{p}\left(B_{-}\right)$. In the first case (3.6) implies $m_{+}(\lambda)=m_{-}(\lambda)$ and hence $\lambda$ is a zero of $m_{+}-m_{-}$. In the second case $\lambda$ is a pole of both $m_{+}$and $m_{-}$. Conversely, let $h_{\lambda} \in \mathfrak{D}_{+}$and $k_{\lambda} \in \mathfrak{D}_{-}$be nontrivial solutions of (3.5) which satisfy $h_{\lambda}(c)=k_{\lambda}(c)$. If $\lambda$ is a zero of $m_{+}-m_{-}$then $\lambda \in \rho\left(B_{+}\right) \cap \rho\left(B_{-}\right)$and $h_{\lambda}(c)=k_{\lambda}(c) \neq 0$, so that the assumption $m_{+}(\lambda)-m_{-}(\lambda)=0$ implies the second condition in (3.6). Therefore $f_{\lambda}=h_{\lambda} \oplus k_{\lambda}$ belongs to $\mathfrak{D}$ and is an eigenfunction of $A$ corresponding to $\lambda$. If $\lambda$ is a pole of $m_{+}$and of $m_{-}$, then $\lambda \in \sigma_{p}\left(B_{+}\right) \cap \sigma_{p}\left(B_{-}\right)$and hence the nontrivial solutions $h_{\lambda} \in \mathfrak{D}_{+}$and $k_{\lambda} \in \mathfrak{D}_{-}$of (3.5) satisfy $h_{\lambda}(c)=k_{\lambda}(c)=0$ and $\left(p_{+} h_{\lambda}^{\prime}\right)(c) \neq 0$ and $\left(p_{-} k_{\lambda}^{\prime}\right)(c) \neq 0$. Since $h_{\lambda}$ and $k_{\lambda}$ are unique up to a constant multiple, it follows that the function

$$
f_{\lambda}=\left(v h_{\lambda}\right) \oplus k_{\lambda}, \quad \text { where } v=\frac{\left(p_{-} k_{\lambda}^{\prime}\right)(c)}{\left(p_{+} h_{\lambda}^{\prime}\right)(c)},
$$

belongs to $\mathfrak{D}$ and is an eigenfunction of $A$ corresponding to $\lambda$.

As a consequence of the above propositions we obtain a statement on the size of the spectral gap of $J A$ around 0 ; cf. Proposition 2.1. We mention that item (ii) in the next proposition can also be deduced from [28], Behauptung 3, applied to the inverses of $A$ and $J A$. 
Proposition 3.3. Assume that conditions (I) and (II) are satisfied. Then the following statements hold:

(i) If $\min \sigma(A)<\min \sigma_{\mathrm{ess}}(A)$, then $[-\min \sigma(A), \min \sigma(A)] \subset \rho(J A)$;

(ii) If $\min \sigma(A)=\min \sigma_{\text {ess }}(A)$, then $(-\min \sigma(A), \min \sigma(A)) \subset \rho(J A)$.

Proof. Let $0<\lambda_{1}=\min \sigma(A)$. We show first that the inclusion

$$
(-\min \sigma(A), \min \sigma(A)) \subset \rho(J A)
$$

holds under any of the assumptions in (i) and (ii), i.e. $\min \sigma(A) \leq \min \sigma_{\text {ess }}(A)$. In fact, by Lemma 2.2 and Proposition 3.2(i) we have that $m_{+}-m_{-}$is holomorphic and does not vanish on $\left(-\lambda_{1}, \lambda_{1}\right)$. Since $m_{+}$and $-m_{-}$are Nevanlinna functions, it follows that $m_{+}$is increasing and $m_{-}$is decreasing on $\left(-\lambda_{1}, \lambda_{1}\right)$. Thus, the images of $m_{+}$and $m_{-}$of $\left(-\lambda_{1}, \lambda_{1}\right)$ are intervals which do not intersect. Consequently, the images of $m_{+}$and $m_{-}(-\cdot)$ of $\left(-\lambda_{1}, \lambda_{1}\right)$ are also intervals which do not intersect, so that $M$ does not vanish on $\left(-\lambda_{1}, \lambda_{1}\right)$. This, together with Proposition 3.1(i) implies that there are no eigenvalues of $J A$ in $\left(-\lambda_{1}, \lambda_{1}\right)$, which yields (3.7) and hence assertion (ii) has been shown.

In order to prove assertion (i) it remains to verify that $\lambda_{1}$ and $-\lambda_{1}$ are not eigenvalues of $J A$ if $\min \sigma(A)<\min \sigma_{\text {ess }}(A)$ holds. We provide the argument for $\lambda_{1}$; a similar reasoning applies to $-\lambda_{1}$. By Proposition 3.2(i) either $m_{+}\left(\lambda_{1}\right)=m_{-}\left(\lambda_{1}\right)$ or both functions $m_{+}$and $m_{-}$have a pole at $\lambda_{1}$. In the first case we have

$$
M\left(\lambda_{1}\right)=m_{+}\left(\lambda_{1}\right)-m_{-}\left(-\lambda_{1}\right)<m_{+}\left(\lambda_{1}\right)-m_{-}\left(\lambda_{1}\right)=0
$$

since $-m_{-}$is a nonconstant Nevanlinna function which is holomorphic on $\left(-\infty, \lambda_{1}\right]$ ( $m_{-}$is not constant as otherwise $\sigma\left(B_{-}\right)=\emptyset$ ). In particular, $M\left(\lambda_{1}\right) \neq 0$ and hence $\lambda_{1}$ is not an eigenvalue of $J A$ by Proposition 3.1(i). If $m_{+}$and $m_{-}$both have a pole at $\lambda_{1}$ then it follows from the holomorphy of $m_{-}$on $\left(-\infty, \lambda_{1}\right)$ that the function $\lambda \mapsto m_{-}(-\lambda)$ is holomorphic in $\lambda_{1}$ and, hence, $M(\cdot)=m_{+}(\cdot)-m_{-}(-\cdot)$ has a pole at $\lambda_{1}$. Again Proposition 3.1(i) implies $\lambda_{1} \in \rho(J A)$.

Note that under the assumptions in Proposition 3.3 an upper estimate for the spectral gap of $J A$ can be given: If $\min \sigma(A)<\min \sigma_{\text {ess }}(A)$ and the smallest eigenvalue $\lambda_{1}=\min \sigma(A)$ of $A$ is a zero of $m_{+}-m_{-}$, then it can be shown that the largest negative eigenvalue $\lambda_{1,-}(J A)$ and the smallest positive eigenvalue $\lambda_{1,+}(J A)$ (i.e. the endpoints of the spectral gap) of $J A$ satisfy

$$
\min \sigma\left(-B_{-}\right)<\lambda_{1,-}(J A) \text { and } \lambda_{1,+}(J A)<\min \sigma\left(B_{+}\right) .
$$

In the case that $\lambda_{1}$ is a pole of $m_{+}$and $m_{-}$the above estimates hold with $\min \sigma\left(-B_{-}\right)$ and $\min \sigma\left(B_{+}\right)$replaced by the second largest eigenvalue of $-B_{-}$and the second smallest eigenvalue of $B_{+}$if these eigenvalues exist, and by $\min \sigma_{\text {ess }}\left(B_{+}\right)$and $\max \sigma_{\text {ess }}\left(-B_{-}\right)$otherwise.

The next proposition and corollary will play an important role in the proof of our main result in the next section. 
Proposition 3.4. The function $M$ admits the representation

$$
M(\lambda)=\frac{-1}{\alpha+\lambda N(\lambda)},
$$

where $N$ is a Nevanlinna function which is not identically zero on $\mathbb{C}$ and $\alpha$ is a real constant. In particular, $M$ is monotonously increasing (monotonously decreasing) on subintervals of $\mathbb{R}^{+}\left(\mathbb{R}^{-}\right.$, respectively) which belong to its domain of holomorphy.

Proof. Let $\lambda \in \rho(J A) \cap \rho\left(B_{+}\right) \cap \rho\left(-B_{-}\right)$and let $h_{\lambda}, h_{0} \in \mathfrak{D}_{+}, k_{-\lambda}, k_{0} \in \mathfrak{D}_{-}$be the unique functions that satisfy

$$
\ell_{+} h_{\lambda}=\lambda h_{\lambda}, \quad \ell_{+} h_{0}=0, \quad \ell_{-} k_{-\lambda}=-\lambda k_{-\lambda}, \quad \ell_{-} k_{0}=0,
$$

and the conditions

$$
\begin{aligned}
h_{\lambda}(c) & =k_{-\lambda}(c), \quad\left(p_{-} k_{-\lambda}^{\prime}\right)(c)-\left(p_{+} h_{\lambda}^{\prime}\right)(c)=1, \\
h_{0}(c) & =k_{0}(c), \quad\left(p_{-} k_{0}^{\prime}\right)(c)-\left(p_{+} h_{0}^{\prime}\right)(c)=1 .
\end{aligned}
$$

We claim that the functions $f_{\lambda}=h_{\lambda} \oplus k_{-\lambda}$ and $f_{0}=h_{0} \oplus k_{0}$ are related via

$$
f_{\lambda}=f_{0}+\lambda(J A-\lambda)^{-1} f_{0} .
$$

For this observe that $\lambda(J A-\lambda)^{-1} f_{0} \in \mathfrak{D}$ and, hence, $g=f_{0}+\lambda(J A-\lambda)^{-1} f_{0}$ satisfies the same conditions as $f_{0}=h_{0} \oplus k_{0}$ in (3.10). Hence, if we write $g$ in the form $g=h \oplus k$ with $h \in \mathfrak{D}_{+}$and $k \in \mathfrak{D}_{-}$, then we have

$$
h(c)=k(c) \quad \text { and } \quad\left(p_{-} k^{\prime}\right)(c)-\left(p_{+} h^{\prime}\right)(c)=1 .
$$

As $(\tau-\lambda) \lambda(J A-\lambda)^{-1} f_{0}=\lambda f_{0}$ we conclude that $\pm \ell_{ \pm}-\lambda$ applied to the restriction of $\lambda(J A-\lambda)^{-1} f_{0}$ onto $(c, \infty)$ and $(-\infty, c)$ equals $\lambda h_{0}$ and $\lambda k_{0}$, respectively. Therefore

$$
\begin{aligned}
& \left(\ell_{+}-\lambda\right) h=\left(\ell_{+}-\lambda\right) h_{0}+\lambda h_{0}=0, \\
& \left(\ell_{-}+\lambda\right) k=\left(\ell_{-}+\lambda\right) k_{0}-\lambda k_{0}=0,
\end{aligned}
$$

and it follows that $h$ and $k$ satisfy the equations $\ell_{+} h=\lambda h$ and $\ell_{-} k=-\lambda k$. Since the function $f_{\lambda}=h_{\lambda} \oplus k_{-\lambda}$ in (3.9) and (3.10) is unique we obtain (3.11).

From

$$
\begin{aligned}
& M(\lambda)=\frac{\left(p_{+} h_{\lambda}^{\prime}\right)(c)-\left(p_{-} k_{-\lambda}^{\prime}\right)(c)}{f_{\lambda}(c)}=-\frac{1}{f_{\lambda}(c)}, \\
& M(0)=\frac{\left(p_{+} h_{0}^{\prime}\right)(c)-\left(p_{-} k_{0}^{\prime}\right)(c)}{f_{0}(c)}=-\frac{1}{f_{0}(c)} \in \mathbb{R},
\end{aligned}
$$


we conclude $M(\lambda) \neq 0$ for $\lambda \in \rho(J A) \cap \rho\left(B_{+}\right) \cap \rho\left(-B_{-}\right)$and $M(0) \neq 0$. With (2.3) we have

$$
\begin{aligned}
\lambda\left[f_{\lambda}, f_{0}\right]= & \lambda\left(h_{\lambda}, h_{0}\right)_{+}-\lambda\left(k_{-\lambda}, k_{0}\right)_{-} \\
= & \left(\ell_{+} h_{\lambda}, h_{0}\right)_{+}-\left(h_{\lambda}, \ell_{+} h_{0}\right)_{+}+\left(\ell_{-} k_{-\lambda}, k_{0}\right)_{-}-\left(k_{-\lambda}, \ell_{-} k_{0}\right)_{-} \\
= & \left(p_{+} h_{\lambda}^{\prime}\right)(c) \overline{h_{0}(c)}-h_{\lambda}(c) \overline{\left(p_{+} h_{0}^{\prime}\right)(c)}-\left(p_{-} k_{-\lambda}^{\prime}\right)(c) \overline{k_{0}(c)} \\
& +k_{-\lambda}(c) \overline{\left(p_{-} k_{0}^{\prime}\right)(c)} \\
= & f_{\lambda}(c)-\overline{f_{0}(c)} .
\end{aligned}
$$

Thus $-M^{-1}$ admits the representation

$$
-M^{-1}(\lambda)=-M^{-1}(0)+\lambda\left[f_{\lambda}, f_{0}\right]
$$

and with (3.11) and $N(\lambda)=\left[\left(1+\lambda(J A-\lambda)^{-1}\right) f_{0}, f_{0}\right]$ we obtain

$$
-M^{-1}(\lambda)=-M^{-1}(0)+\lambda N(\lambda) .
$$

A simple calculation shows

$$
\operatorname{Im} N(\lambda)=\operatorname{Im} \lambda\left(A(J A-\lambda)^{-1} f_{0},(J A-\lambda)^{-1} f_{0}\right)
$$

and since $A$ is nonnegative by condition (II) it follows that $N$ is a Nevanlinna function, i.e. $M$ admits a representation of the from (3.8) with $\alpha=-M(0)^{-1}$.

Note that $N$ is not equal to zero on real intervals which belong to its domain of holomorphy, as otherwise $N \equiv 0$ and (3.12) imply that $M$ in (3.1) is equal to a constant, so that the Titchmarsh-Weyl coefficients $\lambda \mapsto m_{+}(\lambda)$ and $\lambda \mapsto m_{-}(-\lambda)$ of $B_{+}$and $-B_{-}$differ by a real constant; a contradiction to $\sigma\left(B_{+}\right) \cap \sigma\left(-B_{-}\right)=\emptyset$. Now the remaining statements of Proposition 3.4 follow from the fact that the Nevanlinna function $N$ is monotonously increasing on real intervals which belong to its domain of holomorphy.

Corollary 3.5. In between two consecutive positive (resp. negative) poles $v, v^{\prime}$ of $M$ such that the interval $\left(v, v^{\prime}\right)$ belongs to the domain of holomorphy of $M$ there is a unique zero of $M$. Similarly, in between two consecutive positive (resp. negative) zeros $\eta, \eta^{\prime}$ of $M$ such that $M$ is meromorphic in an open neighbourhood of the interval $\left(\eta, \eta^{\prime}\right)$ there is a unique pole of $M$ in $\left(\eta, \eta^{\prime}\right)$.

The poles of $M$ in $\left[\min \sigma\left(B_{+}\right), \infty\right)$ (resp. $\left.\left(-\infty,-\min \sigma\left(B_{-}\right)\right]\right)$coincide with the poles of $\lambda \mapsto m_{+}(\lambda)$ (resp. $\lambda \mapsto m_{-}(-\lambda)$ ), and hence with the isolated eigenvalues of $B_{+}$(resp. $-B_{-}$). From this we obtain with Corollary 3.5 and Proposition 3.1(i) interlacing results of the positive eigenvalues of $J A$ with respect to the eigenvalues of $B_{+}$and of the negative eigenvalues of $J A$ with respect to the eigenvalues of $-B_{-}$. 
Corollary 3.6. In between any two consecutive isolated eigenvalues of $B_{+}$(resp. $-B_{-}$) in a gap of $\sigma_{\mathrm{ess}}\left(B_{+}\right)$(resp. $\sigma_{\mathrm{ess}}\left(-B_{-}\right)$) there is exactly one isolated eigenvalue of $J A$. Conversely, in between any two consecutive isolated positive (resp. negative) eigenvalues of $J A$ in a gap of $\sigma_{\mathrm{ess}}(\mathrm{J} A)$ there is exactly one isolated eigenvalue of $B_{+}$ (resp. $-B_{-}$).

\section{Eigenvalue estimates in gaps of the essential spectrum}

In this section we prove estimates on the number of eigenvalues of $J A$ in a gap of the essential spectrum. Recall that all eigenvalues of the operators $A, J A, B_{+},-B_{-}$ and, hence, $J B$ are simple. For a selfadjoint or $J$-selfadjoint operator $T$ and a real interval $(a, b)$ such that $(a, b) \cap \sigma_{\text {ess }}(T)=\emptyset$ the number of eigenvalues of $T$ in $(a, b)$ will be denoted by $n_{T}(a, b)$, i.e.

$$
n_{T}(a, b)=\sharp\left\{\lambda \in \sigma_{p}(T): \lambda \in(a, b)\right\} .
$$

The following theorem is the main result of this note. It provides a local estimate on the number of eigenvalues of $J A$ in terms of the number of eigenvalues of $A$ in a gap of the essential spectrum. Recall that by Lemma 2.2 and Proposition 2.3 we have for $0 \leq a<b$

$$
(a, b) \cap \sigma_{\mathrm{ess}}(A)=\varnothing \quad \text { if and only if } \quad((-b,-a) \cup(a, b)) \cap \sigma_{\mathrm{ess}}(J A)=\emptyset .
$$

Theorem 4.1. Assume that conditions (I) and (II) hold for the Sturm-Liouville operator $A$ and let $J A$ be the corresponding indefinite Sturm-Liouville differential operator. For $0 \leq a<b$ such that $(a, b) \cap \sigma_{\mathrm{ess}}(A)=\emptyset$ the estimate

$$
\left|n_{A}(a, b)-\left(n_{J A}(-b,-a)+n_{J A}(a, b)\right)\right| \leq 3
$$

is valid if the corresponding quantities are finite; otherwise

$$
n_{A}(a, b)=\infty \text { if and only if } n_{J A}(-b,-a)+n_{J A}(a, b)=\infty .
$$

Observe that the case $n_{A}(a, b)=\infty$ (and, hence, $n_{J A}(-b,-a)+n_{J A}(a, b)=\infty$ ) can only occur if one or both of the endpoints $a$ and $b$ belong to the essential spectrum of $A$ which implies the following corollary.

Corollary 4.2. Let $A, J A$ and $(a, b)$ be as in Theorem 4.1 and assume, in addition, that $b \in \sigma_{\mathrm{ess}}(A)$, or, equivalently, that $b \in \sigma_{\mathrm{ess}}(J A)$ or $-b \in \sigma_{\mathrm{ess}}(J A)$. Then the eigenvalues of $A$ in $(a, b)$ accumulate to $b$ if and only if the eigenvalues of $J A$ in $(-b,-a) \cup(a, b)$ accumulate to $b$ or $-b$.

Proof of Theorem 4.1. Let $(a, b)$ be as in the theorem and suppose that the number $n_{A}(a, b)$ of eigenvalues of $A$ in $(a, b)$ is finite. Since the eigenvalues of $A$ are all simple, $n_{A}(a, b)$ coincides with $\operatorname{dim} \operatorname{ran} E_{A}(a, b)$ and we conclude from Lemma 2.2(iv) 
that dim $\operatorname{ran} E_{B}(a, b)$ differs at most by one from $n_{A}(a, b)$. Hence the number of eigenvalues $n_{B_{+}}(a, b)+n_{-B_{-}}(-b,-a)$ of $J B=B_{+} \oplus-B_{-}$differs at most by one from $n_{A}(a, b)$ and by Proposition 3.1(ii) the same holds true for the number of poles of the function $M$ in $(-b,-a) \cup(a, b)$. It follows from Corollary 3.5 that $M$ has at least $n_{A}(a, b)-3$ zeros in $(-b,-a) \cup(a, b)$, so that

$$
n_{J A}(-b,-a)+n_{J A}(a, b) \geq n_{A}(a, b)-3
$$

by Proposition 3.1(i). In order to show (4.1) suppose that

$$
n_{J A}(-b,-a)+n_{J A}(a, b)>n_{A}(a, b)+3 .
$$

In this case Proposition 3.1(i) yields that there are more than $n_{A}(a, b)+3$ zeros of $M$ in $(-b,-a) \cup(a, b)$ and hence there are more than $n_{A}(a, b)+1$ poles of $M$ in $(-b,-a) \cup(a, b)$ by Corollary 3.5. On the other hand, by the above reasoning the number of poles of $M$ in $(-b,-a) \cup(a, b)$ differs at most by one from $n_{A}(a, b)$, a contradiction and (4.1) is shown.

From (4.1) it follows that for $a$ (or $b$ ) in the essential spectrum of $A$ the quantity $n_{A}(a, b)$ is finite if and only if the quantity $n_{J A}(-b,-a)+n_{J A}(a, b)$ is finite.

Let us now consider the case where the coefficients $p, q$ and $r$ satisfy some symmetry properties with respect to $c$. For simplicity we assume $c=0$ and for the following we suppose:

(III) The functions $p$ and $q$ are even and $r$ is odd, i.e.

$$
p(x)=p(-x), \quad q(x)=q(-x) \quad \text { and } \quad r(x)=-r(-x) \quad \text { for a.e. } x \in \mathbb{R} .
$$

Obviously, (III) implies for the operators $B_{+}$and $B_{-}$from (2.4)

$$
\sigma\left(B_{+}\right)=\sigma\left(B_{-}\right) \quad \text { and } \quad \sigma_{\text {ess }}\left(B_{+}\right)=\sigma_{\text {ess }}\left(B_{-}\right) .
$$

Together with Proposition 2.3 we conclude

$$
\sigma_{\text {ess }}(J A)=\sigma_{\text {ess }}(A) \cup \sigma_{\text {ess }}(-A) .
$$

Furthermore, if $h_{\lambda} \in \mathfrak{D}_{+}$and $k_{\lambda} \in \mathfrak{D}_{-}$are related via $h_{\lambda}(x)=k_{\lambda}(-x), x \in \mathbb{R}^{+}$, then we have

$$
\ell_{+} h_{\lambda}=\lambda h_{\lambda}, \quad \text { if and only if } \quad \ell_{-} k_{\lambda}=\lambda k_{\lambda} \text {. }
$$

Together with $\left(p_{+} h_{\lambda}^{\prime}\right)(0)=-\left(p_{-} k_{\lambda}^{\prime}\right)(0)$ this implies $m_{+}(\lambda)=-m_{-}(\lambda)$ and it follows that the function $M$ in (3.1) is given by

$$
M(\lambda)=m_{+}(\lambda)+m_{+}(-\lambda) .
$$

Observe that by Proposition 3.1(i) the eigenvalues of $J A$ are symmetric with respect to zero. In particular $n_{J A}(a, b)=n_{J A}(-b,-a)$ in Theorem 4.1. This implies the following statement which is a slight improvement of the estimate (4.1) in Theorem 4.1 if condition (III) holds and $n_{A}(a, b)$ is even. 
Corollary 4.3. Let the assumptions be as in Theorem 4.1 and assume, in addition, that condition (III) is satisfied. If $n_{A}(a, b)$ is even, then the estimates

$$
\left|\frac{1}{2} n_{A}(a, b)-n_{J A}(a, b)\right|=\left|\frac{1}{2} n_{A}(a, b)-n_{J A}(-b,-a)\right| \leq 1
$$

are valid.

The estimates in Theorem 4.1 and Corollary 4.3 will be further improved in Theorem 4.4 below for the case that condition (III) holds and instead of a gap in the essential spectrum we consider the special situation of an interval $(\alpha, \beta)$ with $0 \leq \alpha<\min \sigma(A)<\beta \leq \min \sigma_{\mathrm{ess}}(A)$. It is worth mentioning that the following result for the comparison of the quantities $n_{J A}(\alpha, \beta)$ and $n_{A}(\alpha, \beta)$ is optimal.

Theorem 4.4. Assume that conditions (I), (II), and (III) hold for the Sturm-Liouville operator $A$, that $\min \sigma(A)<\min \sigma_{\mathrm{ess}}(A)$ and let $J A$ be the corresponding indefinite Sturm-Liouville differential operator. For $0 \leq \alpha<\min \sigma(A)<\beta \leq \min \sigma_{\mathrm{ess}}(A)$ the following holds:

$$
n_{J A}(\alpha, \beta)=n_{J A}(-\beta,-\alpha)= \begin{cases}\frac{1}{2} n_{A}(\alpha, \beta) & \text { if } n_{A}(\alpha, \beta) \text { is even } \\ \frac{1}{2}\left(n_{A}(\alpha, \beta) \pm 1\right) & \text { if } n_{A}(\alpha, \beta) \text { is odd }\end{cases}
$$

where one of the quantities $n_{A}(\alpha, \beta), n_{J A}(\alpha, \beta), n_{J A}(-\beta,-\alpha)$ is infinite if and only if all the quantities $n_{A}(\alpha, \beta), n_{J A}(\alpha, \beta), n_{J A}(-\beta,-\alpha)$ are infinite.

In particular, the eigenvalues of $A$ below $\min \sigma_{\mathrm{ess}}(A)$ accumulate to $\min \sigma_{\mathrm{ess}}(A)$ if and only if the eigenvalues of $J A$ in the interval $\left(-\min \sigma_{\mathrm{ess}}(A), \min \sigma_{\mathrm{ess}}(A)\right)$ accumulate to $-\min \sigma_{\mathrm{ess}}(A)$ and to $\min \sigma_{\mathrm{ess}}(A)$.

Observe that the case $n_{A}(\alpha, \beta)=\infty$ (and, hence, $n_{J A}(\alpha, \beta)=n_{J A}(-\beta,-\alpha)=$ $\infty)$ can only occur if the endpoint $\beta$ belongs to the essential spectrum of $A$.

Proof. Let $\lambda_{1}=\min \sigma(A)$ be the smallest eigenvalue of $A$. By Proposition 3.2(i) and (4.2) the isolated eigenvalues of the Sturm-Liouville operator $A$ coincide with the poles and zeros of the function $m_{+}$. Hence $\lambda_{1}$ is either a pole or a zero of $m_{+}$. Since $m_{+}$is a Nevanlinna function the poles and zeros of $m_{+}$in $(\alpha, \beta)$ alternate. Therefore one of the following four cases occurs if $n=n_{A}(\alpha, \beta)<\infty$ :

(i) $n$ is even and $\lambda_{1}$ is a pole of $m_{+}$;

(ii) $n$ is even and $\lambda_{1}$ is a zero of $m_{+}$;

(iii) $n$ is odd and $\lambda_{1}$ is a pole of $m_{+}$;

(iv) $n$ is odd and $\lambda_{1}$ is a zero of $m_{+}$.

In case (i) the function $m_{+}$has $\frac{n}{2}$ poles and $\frac{n}{2}$ zeros in $(\alpha, \beta)$. Moreover, the largest eigenvalue $\lambda_{n}$ of $A$ in $(\alpha, \beta)$ is a zero of $m_{+}$and hence $m_{+}$is positive on $\left(-\infty, \lambda_{1}\right) \cup$ $\left(\lambda_{n}, \beta\right)$. The function $M$ has $\frac{n}{2}$ poles in $\left[\lambda_{1}, \lambda_{n-1}\right]$ and it follows from Corollary 3.5 
that there are $\frac{n}{2}-1$ zeros of $M$ in $\left(\lambda_{1}, \lambda_{n-1}\right)$. Since $m_{+}$is positive on $\left(-\infty, \lambda_{1}\right)$ and $m_{+}\left(\lambda_{n}\right)=0$ it follows that $M$ has also one zero in $\left(\lambda_{n-1}, \lambda_{n}\right)$, and is positive on $\left(\alpha, \lambda_{1}\right)$ and $\left[\lambda_{n}, \beta\right)$. Now Proposition 3.1(i) implies $n_{J A}(\alpha, \beta)=\frac{n}{2}=\frac{1}{2} n_{A}(\alpha, \beta)$ and by symmetry also $n_{J A}(-\beta,-\alpha)=\frac{n}{2}=\frac{1}{2} n_{A}(\alpha, \beta)$. The simple modifications of this argument for case (ii) are left to the reader.

In case (iii) the function $m_{+}$has $\frac{1}{2}(n+1)$ poles and $\frac{1}{2}(n-1)$ zeros in $(\alpha, \beta)$. Moreover, $m_{+}$is positive on $\left(-\infty, \lambda_{1}\right)$ and since the largest eigenvalue $\lambda_{n}$ of $A$ in $(\alpha, \beta)$ is a pole $m_{+}$is negative on $\left(\lambda_{n}, \beta\right)$. The function $M$ has $\frac{1}{2}(n+1)$ poles in $\left[\lambda_{1}, \lambda_{n}\right]$ and it follows from Corollary 3.5 that there are $\frac{1}{2}(n-1)$ zeros of $M$ in $\left(\lambda_{1}, \lambda_{n}\right)$. Furthermore, since $m_{+}$is positive on $\left(-\infty, \lambda_{1}\right)$ and negative on $\left(\lambda_{n}, \beta\right)$ there may be one more zero of $M$ in $\left(\lambda_{n}, \beta\right)$. Now Proposition 3.1(i) implies $n_{J A}(\alpha, \beta)=$ $\frac{1}{2}(n \pm 1)=\frac{1}{2}\left(n_{A}(\alpha, \beta) \pm 1\right)$ and by symmetry also $n_{J A}(-\beta,-\alpha)=\frac{1}{2}(n \pm 1)=$ $\frac{1}{2}\left(n_{A}(\alpha, \beta) \pm 1\right)$. The simple modifications of this argument for case (iv) are left to the reader. Relation (4.3) is proved.

From (4.3) it follows also that for $\beta$ in the essential spectrum of $A$ the quantity $n_{A}(\alpha, \beta)$ is finite if and only if the quantities $n_{J A}(\alpha, \beta)$ and $n_{J A}(-\beta,-\alpha)$ are finite.

The next proposition on the interlacing properties of the eigenvalues of $J A$ with respect to the eigenvalues of $A$ can be shown with the same methods as Theorem 4.4. If $(a, b)$ is a gap in $\sigma_{\text {ess }}(A)$ we denote by $\left(\lambda_{k}\right)$ the eigenvalues of $A$ in increasing order, where $k=1, \ldots, n_{A}(a, b)$ if $n_{A}(a, b)$ is finite, $k \in \mathbb{N}(k \in-\mathbb{N})$ if the eigenvalues accumulate to $b$ (resp. $a$ ), and $k \in \mathbb{Z}$ if both endpoints $a$ and $b$ are accumulation points of eigenvalues of $A$.

Proposition 4.5. Assume that conditions (I), (II), and (III) hold for the SturmLiouville operator $A$ and let JA be the corresponding indefinite Sturm-Liouville differential operator. Let $(a, b) \cap \sigma_{\mathrm{ess}}(A)=\emptyset$ and denote by $\left(\lambda_{k}\right)$ the eigenvalues of $A$ in $(a, b)$ in increasing order. Then exactly one of the following statements hold:

(i) each interval $\left(\lambda_{2 k-1}, \lambda_{2 k}\right)$ contains exactly one eigenvalue of $J A$ and each interval $\left[\lambda_{2 k}, \lambda_{2 k+1}\right]$ belongs to $\rho(J A)$;

(ii) each interval $\left(\lambda_{2 k}, \lambda_{2 k+1}\right)$ contains exactly one eigenvalue of $J A$ and each interval $\left[\lambda_{2 k-1}, \lambda_{2 k}\right]$ belongs to $\rho(J A)$.

Furthermore, in the case $a<\lambda_{1}=\min \sigma(A)<b \leq \min \sigma_{\mathrm{ess}}(A)$ statement $(i)$ holds, that is, for the positive eigenvalues $\lambda_{k}(J A)$ of $J A$ ordered in an increasing way we have

$$
\lambda_{k}(J A) \in\left(\lambda_{2 k-1}, \lambda_{2 k}\right), \quad k=1,2, \ldots
$$

\section{Examples}

In this section some applications and examples illustrating the results in the previous section are presented. We start with a variant of Kneser's classical oscillation result 
in the context of indefinite Sturm-Liouville operators. As a second application a periodic problem is treated and in a third explicit example the number of eigenvalues of the indefinite operator is computed for a particularly simple potential.

5.1. Kneser's result for left-definite Sturm-Liouville operators. In this first example accumulation of the eigenvalues of $J A$ to the essential spectrum is studied with the help of Kneser's classical result from [19], see also [11], Corollary XIII.7.57, and [12], [13], [23], [24], [29] for possible generalizations. Here, for simplicity, let $r(x)=\operatorname{sgn}(x), p(x)=1$, and assume that $q>0$ admits the positive limits

$$
0<q_{\infty}=\lim _{x \rightarrow+\infty} q(x)=\lim _{x \rightarrow-\infty} q(x) .
$$

Clearly, condition (I) holds with $c=0$ and by well-known results (see, e.g., [29], Theorem 6.3) the maximal Sturm-Liouville operator $A f=-f^{\prime \prime}+q f, f \in \mathfrak{D}$, satisfies condition (II). Here we have $\sigma_{\text {ess }}\left(B_{ \pm}\right)=\left[q_{\infty}, \infty\right)$ and therefore

$$
\sigma_{\text {ess }}(A)=\left[q_{\infty}, \infty\right) .
$$

By Propositions 2.1 and 2.3 the essential spectrum of the $J$-selfadjoint indefinite Sturm-Liouville operator $J A f=\operatorname{sgn}\left(-f^{\prime \prime}+q f\right), f \in \mathfrak{D}$, is then given by

$$
\sigma_{\text {ess }}(J A)=\left(-\infty,-q_{\infty}\right] \cup\left[q_{\infty}, \infty\right) .
$$

Let us now make use of Kneser's criterion: If

$$
\limsup _{x \rightarrow \infty} x^{2}\left(q(x)-q_{\infty}\right)<-\frac{1}{4} \quad \text { or } \quad \limsup _{x \rightarrow-\infty} x^{2}\left(q(x)-q_{\infty}\right)<-\frac{1}{4}
$$

holds, then there are infinitely many eigenvalues of $B_{+}$or $B_{-}$, respectively, below their essential spectrum and hence also the eigenvalues of $A$ accumulate to $\min \sigma_{\text {ess }}(A)$. By Theorem 4.1 there are infinitely many eigenvalues of $J A$ in the corresponding gap $\left(-q_{\infty}, q_{\infty}\right)$ in $\sigma_{\text {ess }}(J A)$. In the present situation it follows also that the eigenvalues of $J A$ in $\left(-q_{\infty}, q_{\infty}\right)$ accumulate to $q_{\infty}\left(-q_{\infty}\right)$ if the first (second, respectively) condition in (5.1) holds.

Similarly, if instead of (5.1) we have

$$
\liminf _{x \rightarrow \infty} x^{2}\left(q(x)-q_{\infty}\right)>-\frac{1}{4} \text { and } \liminf _{x \rightarrow-\infty} x^{2}\left(q(x)-q_{\infty}\right)>-\frac{1}{4},
$$

then there are only finitely many eigenvalues of $B_{+}$and $B_{-}$below their essential spectrum and hence there are also only finitely many eigenvalues of $A$ below $\min \sigma_{\text {ess }}(A)$. In this situation Theorem 4.1 implies that $J A$ has only finitely many eigenvalues in the corresponding gap around zero and their total number in $\left(-q_{\infty}, q_{\infty}\right)$ differs at most by three of the number of eigenvalues of $A$ below $q_{\infty}=\min \sigma_{\text {ess }}(A)$. 
5.2. Periodic operators. Suppose that the coefficients $|r|, p$ and $q$ of the definite Sturm-Liouville expression $\ell$ are $\gamma$-periodic for some $\gamma>0$ and assume that essinf $q /|r|$ is positive as well as $r$ satisfies condition (I). Then condition (II) is satisfied for the corresponding maximal operator $A$ in $L_{|r|}^{2}(\mathbb{R})$. Furthermore, let $\lambda_{1}<\lambda_{2} \leq \lambda_{3} \leq \ldots$ be the eigenvalues of the selfadjoint operator associated with $\ell$ restricted to functions in $L_{|r|}^{2}(0, \gamma)$ with the boundary conditions

$$
\left(\begin{array}{c}
f(0) \\
\left(p f^{\prime}\right)(0)
\end{array}\right)=\left(\begin{array}{c}
f(\gamma) \\
\left(p f^{\prime}\right)(\gamma)
\end{array}\right)
$$

and let $\mu_{1} \leq \mu_{2} \leq \mu_{3} \leq \cdots$ be the eigenvalues of the selfadjoint operator associated with $\ell$ restricted to functions in $L_{|r|}^{2}(0, \gamma)$ with the boundary conditions

$$
\left(\begin{array}{c}
f(0) \\
\left(p f^{\prime}\right)(0)
\end{array}\right)=-\left(\begin{array}{c}
f(\gamma) \\
\left(p f^{\prime}\right)(\gamma)
\end{array}\right)
$$

Then $0<\lambda_{1}<\mu_{1} \leq \mu_{2}<\lambda_{2} \leq \lambda_{3}<\mu_{3} \ldots$ and it is well-known that

$$
\sigma(A)=\sigma_{\mathrm{ess}}(A)=\left[\lambda_{1}, \mu_{1}\right] \cup\left[\mu_{2}, \lambda_{2}\right] \cup\left[\lambda_{3}, \mu_{3}\right] \ldots
$$

holds, see, e.g., [29], $\S 12$. Here it follows that also $\sigma_{\text {ess }}\left(B_{+}\right)=\sigma_{\text {ess }}\left(B_{-}\right)=\sigma_{\text {ess }}(A)$ holds, and therefore by Proposition 2.3 the essential spectrum $\sigma_{\text {ess }}(J A)$ of $J A$ has a band structure, is symmetric with respect to 0 and is given by

$$
\ldots\left[-\mu_{3},-\lambda_{3}\right] \cup\left[-\lambda_{2},-\mu_{2}\right] \cup\left[-\mu_{1},-\lambda_{1}\right] \cup\left[\lambda_{1}, \mu_{1}\right] \cup\left[\mu_{2}, \lambda_{2}\right] \cup\left[\lambda_{3}, \mu_{3}\right] \ldots
$$

Since $A$ has no eigenvalues in the (possible) gaps $\left(\mu_{1}, \mu_{2}\right),\left(\lambda_{2}, \lambda_{3}\right),\left(\mu_{3}, \mu_{4}\right), \ldots$, of $\sigma_{\mathrm{ess}}(A)$ we conclude from Theorem 4.1 that each of the sets

$$
\left(-\mu_{2},-\mu_{1}\right) \cup\left(\mu_{1}, \mu_{2}\right), \quad\left(-\lambda_{3},-\lambda_{2}\right) \cup\left(\lambda_{2}, \lambda_{3}\right), \quad\left(-\mu_{4},-\mu_{3}\right) \cup\left(\mu_{3}, \mu_{4}\right), \ldots
$$

contains at most 3 eigenvalues of the indefinite Sturm-Liouville operator $J A$. Note that by Proposition 3.3 we have $\left(-\lambda_{1}, \lambda_{1}\right) \subset \rho(J A)$. Furthermore, if the coefficients $r, p$, and $q$ satisfy the symmetry condition (III), then Corollary 4.3 implies that in each of the (possible) gaps

$$
\ldots\left(-\mu_{4},-\mu_{3}\right),\left(-\lambda_{3},-\lambda_{2}\right),\left(-\mu_{2},-\mu_{1}\right),\left(\mu_{1}, \mu_{2}\right),\left(\lambda_{2}, \lambda_{3}\right),\left(\mu_{3}, \mu_{4}\right), \ldots
$$

of $\sigma_{\mathrm{ess}}(J A)$ there is at most one eigenvalue.

5.3. A solvable problem with a hyperbolic cosine potential. As an explicit example consider the situation $r(x)=\operatorname{sgn} x, p(x)=1$ and

$$
q(x)=(\kappa+1)^{2}-\frac{\kappa(\kappa+1)}{\cosh ^{2}(x)} \quad \text { for some } \kappa \in \mathbb{N} .
$$


Obviously, conditions (I) and (III) are satisfied. Moreover, $q(x) \geq \kappa+1$ and $\lim _{|x| \rightarrow \infty} q(x)=(\kappa+1)^{2}$ imply that for the corresponding maximal operator $A$ we have $\min \sigma(A) \geq \kappa+1$ and $\sigma_{\text {ess }}(A)=\left[(\kappa+1)^{2}, \infty\right)$. In particular, condition (II) is also fulfilled. It is known (see, e.g., [14]), that the operator $A$ has precisely $\kappa$ eigenvalues in the interval $\left(\kappa+1,(\kappa+1)^{2}\right)$. Therefore, the essential spectrum of the corresponding indefinite Sturm-Liouville operator $J A$ is given by

$$
\sigma_{\text {ess }}(J A)=\left(-\infty,-(\kappa+1)^{2}\right] \cup\left[(\kappa+1)^{2}, \infty\right)
$$

and according to Theorem 4.4 the operator $J A$ has $\frac{\kappa}{2}$ eigenvalues in the interval $\left(\kappa+1,(\kappa+1)^{2}\right)$ if $\kappa$ is even and $\frac{\kappa \pm 1}{2}$ eigenvalues if $\kappa$ is odd. The same holds for the interval $\left(-(\kappa+1)^{2},-(\kappa+1)\right)$; cf. Theorem 4.4. Note that by Proposition 3.3 $\kappa+1$ and $-(\kappa+1)$ are no eigenvalues of $J A$.

\section{References}

[1] T. Ya. Azizov and I. S. Iokhvidov, Linear operators in spaces with an indefinite metric. John Wiley Sons, Chichester, 1989. MR 1033489 Zbl 0714.47028

[2] J. Behrndt, On the spectral theory of singular indefinite Sturm-Liouville operators. J. Math. Anal. Appl. 334 (2007), 1439-1449. MR 2338672 Zbl 1126.34052

[3] J. Behrndt, Q. Katatbeh, and C. Trunk, Non-real eigenvalues of singular indefinite Sturm-Liouville operators. Proc. Amer. Math. Soc. 137 (2009), 3797-3806. MR 2529889 Zbl 1182.47036

[4] J. Behrndt and F. Philipp, Spectral analysis of singular ordinary differential operators with indefinite weights. J. Differ. Equations 248 (2010), 2015-2037. MR 2595713 Zbl 1197.34167

[5] J. Behrndt and C. Trunk, On the negative squares of indefinite Sturm-Liouville operators. J. Differ. Equations 238 (2007), 491-519. MR 2341434 Zbl 1123.47033

[6] M. Sh. Birman and M. Z. Solomjak, Spectral theory of self-adjoint operators in Hilbert space. Kluwer Academic Publishers, Dordrecht etc., 1987. Zbl 0744.47017

[7] J. Bognar, Indefinite inner product spaces. Springer Verlag, New York etc., 1974. MR 0467261 Zbl 0286.46028

[8] B. Ćurgus and H. Langer, A Krein space approach to symmetric ordinary differential operators with an indefinite weight function. J. Differ. Equations 79 (1989), 31-61. MR 0997608 Zbl 0693.34020

[9] B. Curgus and B. Najman, The operator $\operatorname{sgn}(x) \frac{d^{2}}{d x^{2}}$ is similar to a selfadjoint operator in $L^{2}(\mathbb{R})$. Proc. Amer. Math. Soc. 123 (1995), 1125-1128. MR 1223513 Zbl 0835.47021

[10] K. Daho and H. Langer, Sturm-Liouville operators with an indefinite weight function. Proc. R. Soc. Edinb., Sect. A 78 (1977/78), 161-191. MR 0492490 Zbl 0372.34018

[11] N. Dunford and J. T. Schwartz, Linear operators. Part II: Spectral theory. Self adjoint operators in Hilbert space. John Wiley \& Sons, New York etc., 1963. MR 0188745 Zbl 0128.34803 Zbl 0635.47002 
[12] F. Gesztesy, B. Simon, and G. Teschl, Zeros of the Wronskian and renormalized oscillation theory. Amer. J. Math. 118 (1996), 571-594. MR 1393260 Zbl 0858.47027

[13] F. Gesztesy and M. Ünal, Perturbative oscillation criteria and Hardy-type inequalities. Math. Nachr. 189 (1998), 121-144. MR 1492926 Zbl 0903.34030

[14] R. L. Hall, Square-well representation for potentials in quantum mechanics. J. Math. Phys. 33 (1992), 3472-3476. MR 1182920 Zbl 0762.35094

[15] I. M. Karabash, A. S. Kostenko, and M. M. Malamud, The similarity problem for $J$-nonnegative Sturm-Liouville operators. J. Differ. Equations 246 (2009), 964-997. MR 2474582 Zbl 1165.34012

[16] I. M. Karabash and M. M. Malamud, Indefinite Sturm-Liouville operators $(\operatorname{sgn} x)\left(-\frac{d^{2}}{d x^{2}}+q(x)\right)$ with finite-zone potentials. Oper. Matrices 1 (2007), 301-368. MR 2344680 Zbl 1146.47032

[17] I. M. Karabash and C. Trunk, Spectral properties of singular Sturm-Liouville operators with indefinite weight sgn x. Proc. R. Soc. Edinb., Sect. A 139 (2009), 483-503. MR 2506783 Zbl 1189.34159

[18] I. S. Kac and M. G. Krein, $R$-functions - analytic functions mapping the upper halfplane into itself. Appendix I to the russian edition of F. V. Atkinson, Discrete and continuous boundary problems. Mir, Moscow, 1968. English transl. of the Appendix: Am. Math. Soc., Translat., II. Ser. 103 (1974), 1-18. Zbl 0291.34016

[19] A. Kneser, Untersuchungen über die reellen Nullstellen der Integrale linearer Differentialgleichungen. Math. Ann. 42 (1893), 409-435. MR 1510784 JFM 25.0522.01

[20] Q. Kong, M. Möller, H. Wu, and A. Zettl, Indefinite Sturm-Liouville problems. Proc. R. Soc. Edinb., Sect. A 133 (2003), 639-652. MR 1983691 Zbl 1056.34038

[21] Q. Kong, H. Wu, and A. Zettl, Singular left-definite Sturm-Liouville problems. J. Differ. Equations 206 (2004), 1-29. MR 2093917 Zbl 1058.34029

[22] A. Kostenko, Similarity problem for indefinite Sturm-Liouville operators with periodic coefficients. Preprint 2010. arXiv:1004.3991

[23] H. Krüger and G. Teschl, Effective Prüfer angles and relative oscillation criteria. J. Differ. Equations 245 (2008), 3823-3848. MR 2462706 Zbl 1167.34009

[24] H. Krüger and G. Teschl, Relative oscillation theory, weighted zeros of the Wronskian, and the spectral shift function. Commun. Math. Phys. 287 (2009), 613-640. MR 2481752 Zbl 1186.47009

[25] H. Langer, Spectral functions of definitizable operators in Krein spaces. In D. Butkovic, D. H. Kraljevic, and S. Kurepa, Svetozar (eds.), Functional analysis: Proceedings of a Conference Held at Dubrovnik, Yugoslavia, November 2-14, 1981. Springer Verlag, Berlin etc., 1982, 1-46. MR 0672791 Zbl 0511.47023

[26] M. Marletta and A. Zettl, Floquet theory for left-definite Sturm-Liouville problems. J. Math. Anal. Appl. 305 (2005), 477-482. MR 2130715 Zbl 1074.34030

[27] R. Rosenberger, A new characterization of the Friedrichs extension of semibounded Sturm-Liouville operators. J. Lond. Math. Soc., II. Ser. 31 (1985), 501-510. MR 0812779 Zbl 0615.34019

[28] B. Textorius, Minimaxprinzipe zur Bestimmung der Eigenwerte $J$-nichtnegativer Operatoren. Math. Scand. 35 (1974), 105-114. MR 0370259 Zbl 0328.47016 
[29] J. Weidmann, Spectral theory of ordinary differential operators. Springer Verlag, Berlin etc., 1987. MR 0923320 Zbl 0647.47052

[30] A. Zettl, Sturm-Liouville theory. Amer. Math. Soc., Providence (RI), 2005. MR 2170950 Zbl 1103.34001

Received December 16, 2010; revised March 21, 2011

Jussi Behrndt, Institut für Numerische Mathematik, Technische Universität Graz, Steyrergasse 30, A-8010 Graz, Austria

E-mail: behrndt@tugraz.at

Roland Möws, Institut für Mathematik,

Fakultät für Mathematik und Naturwissenschaften, Technische Universität Ilmenau, Postfach 1005 65, D-98684 Ilmenau, Germany

E-mail: roland.moews@tu-ilmenau.de

Carsten Trunk, Institut für Mathematik,

Fakultät für Mathematik und Naturwissenschaften, Technische Universität Ilmenau, Postfach 1005 65, D-98684 Ilmenau, Germany

E-mail: carsten.trunk@tu-ilmenau.de 